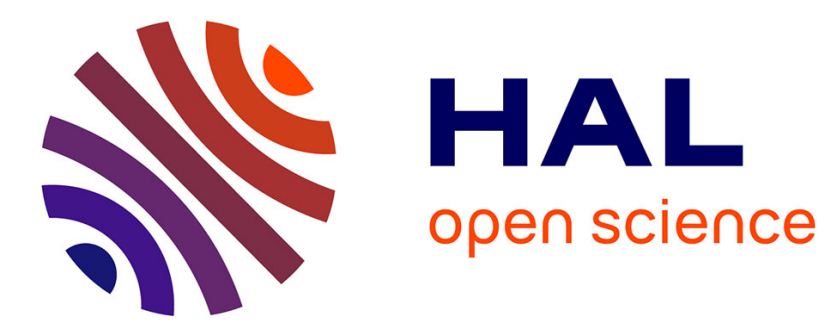

\title{
Bubble-based acoustic micropropulsors: active surfaces and mixers
}

Nicolas A Bertin, Tamsin A Spelman, Thomas Combriat, Hervé Hue, Olivier Stéphan, Eric Lauga, Philippe Marmottant

\section{To cite this version:}

Nicolas A Bertin, Tamsin A Spelman, Thomas Combriat, Hervé Hue, Olivier Stéphan, et al.. Bubblebased acoustic micropropulsors: active surfaces and mixers. Lab on a Chip, 2017, 17 (8), pp.1515 1528. 10.1039/c7lc00240h . hal-01640096

\section{HAL Id: hal-01640096 https://hal.science/hal-01640096}

Submitted on 15 Jul 2019

HAL is a multi-disciplinary open access archive for the deposit and dissemination of scientific research documents, whether they are published or not. The documents may come from teaching and research institutions in France or abroad, or from public or private research centers.
L'archive ouverte pluridisciplinaire HAL, est destinée au dépôt et à la diffusion de documents scientifiques de niveau recherche, publiés ou non, émanant des établissements d'enseignement et de recherche français ou étrangers, des laboratoires publics ou privés. 


\title{
Bubble-based acoustic micropropulsors: active surfaces and mixers
}

\author{
Nicolas Bertin, ${ }^{a}$ Tamsin A. Spelman, ${ }^{c}$ Thomas Combriat,${ }^{b}$ Hervé Hue,${ }^{b}$ Olivier Stéphan, ${ }^{b}$ Éric Lauga ${ }^{c}$ and Philippe \\ Marmottant ${ }^{b}$ \\ Acoustic micropropulsors present great potential for microfluidic applications. The propulsion is based on encapsulated $20 \mu \mathrm{m}$ \\ bubbles excited by a contacless ultrasonic transducer. The vibrating bubbles then generate a powerful streaming flow, with \\ speeds $1-100 \mathrm{~mm} / \mathrm{s}$ in water, through the action of viscous stresses. In this paper we introduce a full toolbox of micropropulsors \\ using a versatile three-dimensional (3D) microfabrication setup. Doublets and triplets of propulsors are introduced, and the \\ flows they generate are predicted by a theoretical hydrodynamic model. We then introduce whole surfaces covered with \\ propulsors, which we term active surfaces. These surfaces are excited by a single ultrasonic wave, can generate collective \\ flows and may be harnessed for mixing purposes. Several patterns of propulsors are tested, and the flows produced by the \\ two most efficient mixers are predicted by a simple theoretical model based on flow singularities. In particular, the vortices \\ generated by the most efficient pattern, an L-shaped mixer, are analysed in detail.
}

\section{Introduction}

Microbubbles are powerful active elements ${ }^{36}$, and have been used in a variety of applied setups, including particle transport ${ }^{15}$, manipulation ${ }^{27}$ and for mixing purposes $3,4,8,41$. These applications harness the powerful flows generated by remotely excited $4,8,18,26,29,40,41$ microbubbles. However, one weakness of microbubbles is their short life span. Indeed a free micron-sized air bubble dissolves in water at room temperature in tens of seconds. In a previous study ${ }^{8}$, we overcame this issue by proposing a new type of acoustic micropropulsor, namely armoured microbubbles (AMBs). An AMB is composed of a capsule, which is a 3D printed hollow partial sphere fabricated on top of a short pole, inside which a microbubble can be caught when immersed in water (see sketch in fig. 1a and experimental image in fig. 2a). Recently, stereolithographic 3D printers (also called SLA printers) were used to build channels, valves and pumps ${ }^{7,16}$, and to fabricate electrospray sources in millifluidics ${ }^{31}$. In order to print the capsules, our setup uses 2-photon absorption lithography to reach sub-micron fabrication precision, allowing us to manufacture capsules on the $10 \mu \mathrm{m}$ scale. We fabricate capsules with opening diameter at most half of the capsule's overall diameter, due to shorter bubble lifespan at larger opening diameters. Under ultrasound, the free surface of the bubble near the opening of the capsule oscillates, generating a powerful flow via acoustic streaming. We can predict this flow with a detailed 3D hydrodynamic model and also predict the resonance frequencies of the bubbles ${ }^{8}$.

Having previously performed a detailed analysis for an isolated $\mathrm{AMB}^{8}$, we consider in this article the variety of flows achievable by multiple AMBs. We first consider multipropulsor compounds,

\footnotetext{
${ }^{a}$ Univ. Grenoble Alpes and CNRS, UMR 5588 LIPhy, F-38402 Grenoble, France. E-mail: n.m.bertin@gmail.com

${ }^{b}$ Univ. Grenoble Alpes and CNRS, UMR 5588 LIPhy, F-38402 Grenoble, France.

c DAMTP, University of Cambridge, Cambridge CB3 OWA, United Kingdom.
}

where multiple AMBs are physically attached together, and then address active surfaces, where multiple AMBs are placed in a pattern short distances apart. Virtually any arrangement of AMBs may be fabricated using our method and we demonstrate the variety of flows achievable. We consider below specific AMB compounds such as double propulsors, which produces a wider jet than a single AMB, and triple propulsors, which produce multi-directional flows. Moving on to active surfaces, we see that the flows they produce can display large vortices which, through Taylor-Aris dispersion $^{6,39}$, are able to enhance significantly effective diffusion. This feature, combined with the remote actuation of an active surface, its high adaptability and minimal impact on the base flow when the ultrasound is switched off, make active surfaces a good candidate for a microfluidics mixer.

\section{Methods}

\subsection{Fabrication of capsules and Set-up}

We use a 2-photon absorption microscopic device (Microlight ${ }^{1}$ ) to fabricate hollow capsules of inner diameter $2 r$ with an opening of diameter $2 a$ (see fig. 1a). The resin is OrmoComp ${ }^{\circledR}{ }^{2}$, a biocompatible hybrid polymer used for ultraviolet (UV) imprint and moulding. We prepare a solution of $6 \mathrm{mg}$ 1,3,5-Tris(2-(9ethylcabazyl-3)ethylene)benzene photoinitiator dissolved in $1 \mathrm{ml}$ dichloromethane, and mixed with $1 \mathrm{~g}$ OrmoComp ${ }^{\circledR}$. The resin is only polymerised in the vicinity of the beamwaist of the laser, and the precision is on the order of the $500 \mu \mathrm{W}$ Nd:YAG microchip laser wavelength, $532 \mathrm{~nm}^{24}$. The laser writing speed is estimated at $50 \mu \mathrm{m} / \mathrm{s}$, with $2 \mathrm{~ms}$ laser pulses. The setup is mounted on the epifluorescence port of an inverted microscope.

In the measurements shown below, all capsules have an internal radius $r=9 \mu \mathrm{m}$, and a double wall construction. The distance between the two walls is $1 \mu \mathrm{m}$ so the external radius is $10 \mu \mathrm{m}$. The opening radius is $a=5 \mu \mathrm{m}$ unless otherwise stated. Indeed, we have discovered that the optimum ratio for increasing the bubble 
(a)

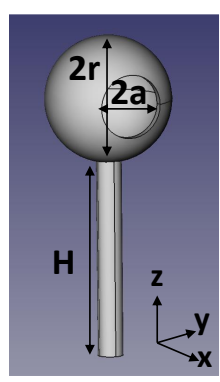

(c)

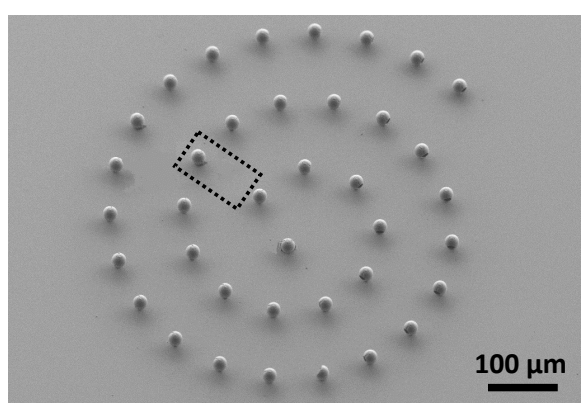

(b)

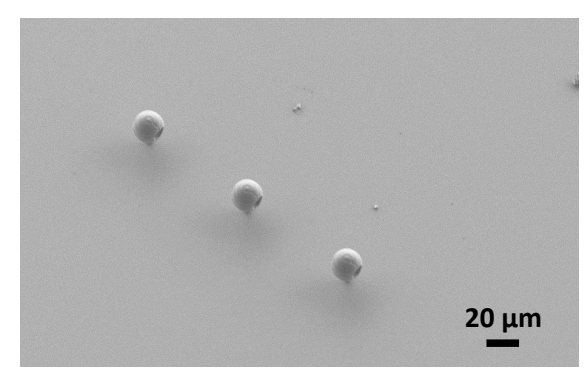

(d)

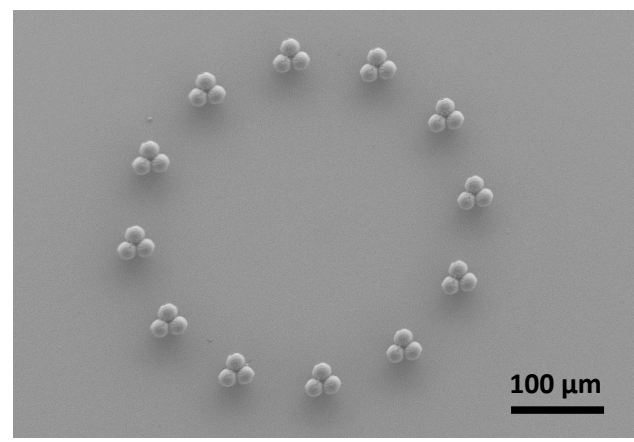

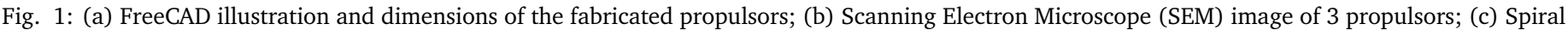
of propulsors (a dust fiber has been edited out of the image next to an AMB, see dashed rectangle); (d) Triple propulsors arrange in a circle pattern.

lifespan is $a / r \approx 0.5$. We build AMBs with $2 \mu$ m radius poles of height $H=10 \mu \mathrm{m}$, which provide more stability during fabrication than the $H=30 \mu \mathrm{m}$ poles considered in earlier work ${ }^{8}$. This is especially important for our heavier multipropulsor compounds. To overcome the difficulty inherent to printing multiple objects, we added an autofocus capability to the setup. The tower and capsule are printed onto a glass coverslip used as a substrate, but at the micron scale, this surface will not be completely flat. The object must be printed on the substrate, and not above, else it will be washed away during rinsing. Therefore at the start of fabrication of a new object, the beamwaist of the laser should be positioned at or just below the surface of the glass coverslip. A lateral movement of the piezoelectric stage of $100 \mu \mathrm{m}$ may result in a vertical shift of approximately 0.5 to $1 \mu \mathrm{m}$ or a tilt angle of the coverslip of 0.3 to $0.6^{\circ}$. We automatically correct for any vertical discrepancy using our autofocus script. It analyses at low laser power the light intensity transmitted at various altitudes, in order to find the exact position of the laser beamwaist relative to the coverslip.

In order to allow us to print a large number of objects in predetermined patterns such as spirals (fig. 1c), circles (fig. 1d), and triangles (fig. 3a), we program a long-range translation stage for a list of positions using a Python script. Since changing the direction of an AMB only requires rotating it around the $z$ axis (see notation in fig. 1a), our patterns include capsules with openings pointing in all possible directions.

The fabrication process is autonomous once the printing area is defined. A propulsor takes approximately $15 \mathrm{~min}$ to print, so 100 propulsors can be fabricated in 28 hours. The only time limitation is due to the resin, which naturally cures over about 48 hours. Scanning Electron Microscope (SEM) observations of the fabricated objects confirmed the reproducibility of the process (see fig. 1b-d).

\subsection{Acoustic streaming flow prediction around individual propulsors and AMB arrays}

We have previously derived a theoretical model for calculating the streaming flow around a single AMB both in free space and near a wall ${ }^{8,35}$. In order to approximate the streaming flow induced by double and triple propulsors, we linearly superpose the streaming flows each of the AMBs produce individually when isolated and close to a wall. Since we are superposing individual solutions which only obey the boundary conditions on their own AMB, this introduces error and the resulting streaming flow approximation is not valid close to where the AMBs meet. Additional errors are added close to the AMBs due to the importance of inertia in the boundary layer close to the AMBs. But outside the boundary layer the streaming flow is a Stokes flow and so a short distance from the AMBs our approximation is valid.

We use a different approximation to calculate the streaming flow around AMB arrays. Indeed, for AMB arrays the length scales of configurations are $O(100 \mu \mathrm{m})$ as opposed to $O(10 \mu \mathrm{m})$ for individual AMBs. Therefore we may approximate the flow field induced by each AMB in free space as its leading-order solution in the far field $^{8}$, namely a Stokeslet (point force), plus its next-order correction of a Stresslet. The Stokeslet strength for an AMB $f_{t}$ was chosen so the flow velocity $\approx 10 \mu \mathrm{m}$ from the Stokeslet was of the order of $1-100 \mathrm{~mm} / \mathrm{s}$, the range of velocities observed in front of an AMB experimentally. The flow field of a Stresslet is determined by a tensor, which for an AMB pointing in the $x$ direction modeled at leading order by a Stokeslet of strength $f_{t}$ in the $x$ direction, is 

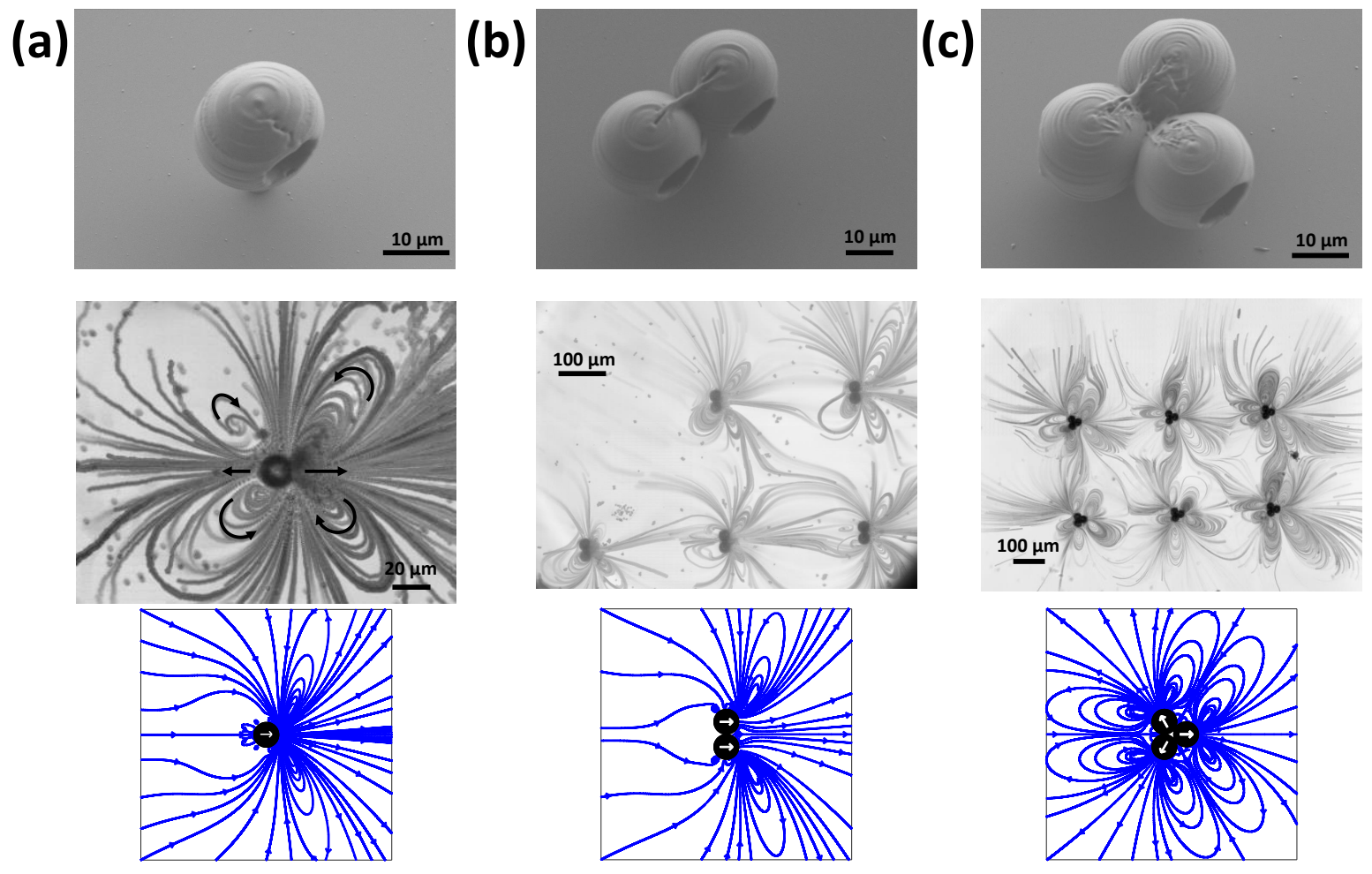

Fig. 2: Vortices generated by identical propulsors ( $r=9 \mu \mathrm{m}, a=5 \mu \mathrm{m}, H=10 \mu \mathrm{m}$ ) arranged as: (a) Single propulsors; (b) Double propulsors; (c) Triple propulsors. Top line: SEM image, middle line: streamlines obtained by the superposition of tracer positions, bottom line: theoretical predictions of the streamlines.

given by

$$
f_{t}\left(\begin{array}{ccc}
-8 / 3 & 0 & 0 \\
0 & 4 / 3 & 0 \\
0 & 0 & 4 / 3
\end{array}\right) \text {. }
$$

This form and strength of the Stresslet is given by the full solution of the flow around the $\mathrm{AMB}^{8,35}$. Both the Stokeslet and Stresslet are singular solutions; in order to make the flow field finite everywhere we then mathematically regularise the Stokeslet and Stresslet. Additionally, to model the AMBs near one wall we add the images of the Stokeslet and Stresslet. More explicitly, near a flat plate each AMB is modelled using the regularised version of the well known Blake solution for a Stokeslet above a no-slip wall 5,9,12 plus the Stresslet with its known solution near one wall ${ }^{5,12}$. We then linearly superpose the flow fields generated by each AMB individually near one wall to obtain the flow field induced by the whole AMB array. If there is a base flow present, we linearly add that contribution to the AMB array flow field.

To model AMB arrays in a channel (i.e. sandwiched between two close walls and with two side walls), we use a slightly different approach. We approximate each AMB by its leading order Stokeslet of strength $f_{t}$ only (so we ignore the Stresslet correction). Between two walls a Stokeslet has a well known solution ${ }^{25}$. To obtain the flow field in a vertical cross section of the channel, we linearly superpose this individual solution for each AMB between two walls.

We then use the fact that the solution for a Stokeslet parallel to two confining walls has a vertical component of velocity which decays exponentially, whereas the planar component of the velocity decays as a power law. Therefore in the far field, the vertical velocity component is negligible. The horizontal velocity is given by

$$
\underline{u}(x, y)=\frac{4}{h^{2}} z(h-z) \underline{u}(x, y),
$$

where $z=0, h$ is the bottom and top plate respectively, and $\underline{\tilde{u}}$ is a 2D Stokes Doublet which, for an AMB pointing in the $x$ direction, is

$$
\tilde{u}_{j}^{1}=-\frac{3 f_{t}}{4 \pi \mu} H\left(1-\frac{H}{h}\right) \frac{1}{\rho^{2}}\left[\frac{1}{2} \delta_{j 1}-\frac{r_{j} r_{1}}{\rho^{2}}\right],
$$

where $\mathrm{j}=1,2, \mu$ is the dynamic viscosity of the fluid, $\left(y_{1}, y_{2}, H\right)$ is the position of the Stokeslet, $\rho=\sqrt{\left(x-y_{1}\right)^{2}+\left(y-y_{2}\right)^{2}}$ is the distance in the plane from the Stokeslet, and $r_{1}=\left(x-y_{1}\right), r_{2}=\left(y-y_{2}\right)$ is the straight line distances from the Stokeslet in the $x$ and $y$ direction.

We see that in the far field the flow is equal to a two-dimensional Stokes Doublet as in Eq.(3) with a magnitude which varies quadratically across the channel as in Eq.(2) ${ }^{25}$. We can then regularise this Stokes Doublet to remove singularities using the technique described in refs. ${ }^{12,13}$. We then linearly superpose this individual solution for each AMB to obtain the total flow field around the AMB array. Note that the result in Eq. (2) shows that the choice of horizontal plane (i.e. the value of $z$ ) only affects the total magnitude of the flow field thus the flow field is structurally the same in every plane (at leading order). When there is a base flow of strength $4 v_{0} / h^{2}$ (for constant $v_{0}$ ), we assume it is a traditional 2D Poiseuille flow with velocity field $\underline{U_{0}}=\left(4 v_{0} / h^{2}\right) z(h-z) e_{y}$, for $e_{y}$ pointing down the channel, and thus has the same quadratic dependence across the channel as the flow generated by the AMB array, so when we linear superpose it the flow is still structurally 
the same in each plane. We note that this model ignores the effects of the side walls since they are $1 \mathrm{~mm}$ apart as opposed to $80 \mu \mathrm{m}$ for the channel height, an accurate assumption when the AMB arrays are placed away from these side walls and the AMBs are weak enough for their effects to not extend across the channel width.

\subsection{Theoretical mixing measure of AMB arrays}

Most theoretical studies of mixing use detailed numerical simulations ${ }^{11,42}$ or scaling arguments ${ }^{19,21}$. Here we present an alternative approach, namely a numerical method applied to a simplified mixer-channel setup. The advantage of our method is that, due to its simplicity, it can analyse hundreds of mixers a day, and is designed to identify groups of good mixers which can then undergo further detailed numerical and experimental study.

We make use of the far-field flow field outlined above for the mixer between two plane walls. Since every plane is structurally the same we focus our analysis on one plane. The regularisation of the Stokes Doublet breaks into a source and sink, but by choosing a regularisation length of half the radius of the AMB these are contained well within the AMB. To prevent fluid unphysically becoming trapped in a sink, we add a $A e^{-B /\left(r-r_{0}\right)^{2}}$ velocity contribution in the direction of the Stokes Doublet within the AMB radius $r_{0}$ (where $A, B$ are constants) to push flow from the sink to the source. The maximum velocity within the AMB is maintained at the same order. This contribution is continuous at all derivatives on the boundary of the AMB and is added only within the AMB. The AMB strength was taken as $0.45 \mathrm{nN}$ with a base flow in the AMB plane of $1.2 \mathrm{~mm} / \mathrm{s}$.

Diffusion will ultimately cause the mixing but its effectiveness will be dependent on the size of the surface area between the two fluids which the mixer will increase. In our plane we consider a section of line $3.2 \mathrm{~mm}$ in length which divides the two fluids. We measure mixing capability with the ratio of the length of line after it has partially passed through the mixer to its initial length. We take our stretch of line starting away from the mixer where the base flow dominates, and thus we can assume that each point on the line follows the same path, only with a time delay. The analysis is terminated when the end of this line element reaches the starting position of the front of the line element, at which point the front of the line element will have well passed out the other side of the mixer (if not caught in a mixer circulation).

\section{Arrangements of propulsors}

\subsection{Individual propulsors: single, doublets or triplets}

Bubbles resonate at a wavelength much greater than their radius $^{30}$. In the case studied here, the $r=9 \mu \mathrm{m} \mathrm{AMB} \mathrm{with} \mathrm{an} a=5$ $\mu \mathrm{m}$ opening resonates at a frequency of $f_{r}=320 \mathrm{kHz}^{8}$, which corresponds to an acoustic wavelength of $\lambda=4.7 \mathrm{~mm}$ in water. The resonance frequency for $a=3 \mu \mathrm{m}$ increases to $f_{r}=510 \mathrm{kHz}$ and for $a=7.5 \mu \mathrm{m}$ it decreases to $f_{r}=160 \mathrm{kHz}$. We verified experimentally by measuring the resonance frequency that, at constant $a$ and increasing $V, f_{r}$ is indeed constant. Since $\lambda \gg r$, we are able to excite a large number of propulsors with the same focused ultrasonic wave (55 propulsors on fig. 3), the acoustic beam width being on (a)

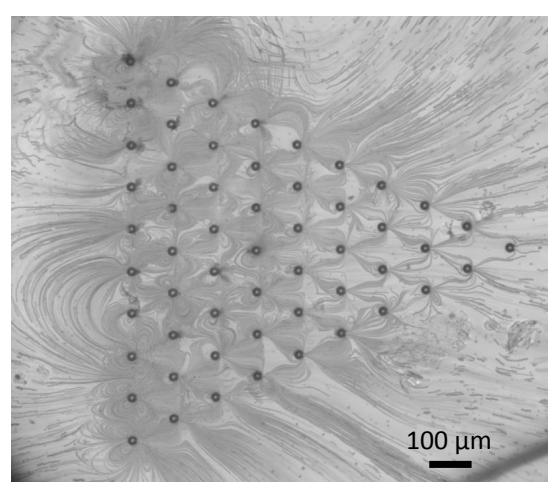

(b)



(c)

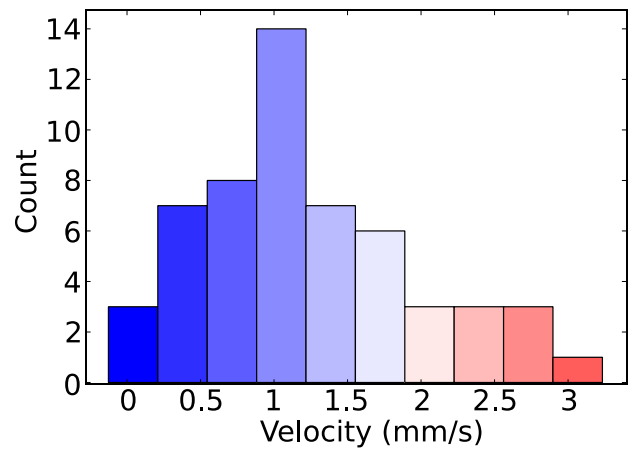

Fig. 3: Triangle of propulsors. (a) Streamlines from a 10 line triangle (propulsors pointing right); (b) PTV analysis of $v_{x}$ showing no additive effect; (c) Histogram of the average of $v_{x}$ inside a 10 by $5 \mu \mathrm{m}$ rectangle placed $5 \mu \mathrm{m}$ in front of each AMB.

the order of $\lambda$. In fig. $2 \mathrm{a}$, the flow generated by a $H=10 \mu \mathrm{m}$ capsule is illustrated, showing two pairs of counter-rotating vortices. The backward, slower pair of vortices is due to a boundary effect absent when $H=30 \mu \mathrm{m}^{8}$. Such a propulsor is able to generate forward flows with mean velocity $v_{\text {mean }}=1-100 \mathrm{~mm} / \mathrm{s}$.

We next take advantage of the combined flow from different AMBs and design multipropulsor compounds. In order to extend the lateral range of the forward flow of a single $A M B$, we first developed a double propulsor. It consists of 2 solidly bound, iden- 
tical, adjacent capsules, mounted on the same $10 \mu \mathrm{m}$ pole. The width of the generated forward liquid jet is doubled as a result, see fig. $2 \mathrm{~b}$ (with similar jet widening also observed at $a=7.5$ ).

We then considered what multipropulsor compound could generate vortices in all directions in the $x-y$ plane, similarly to a free $2 \mathrm{D}$ bubble in a microchannel ${ }^{28,29}$, but protected from dissolution by the capsules. To this end, we developed a triple propulsor consisting of 3 capsules back to back, with openings oriented at $120^{\circ}$ from one another generating 3 pairs of vortices (see fig. 2c). Note that these multi-propulsor compounds have the advantage of having the same resonant frequency as each of the individual propulsors composing it. Both the double and triple propulsor produce flows that can be predicted accurately with our streaming model as shown on fig. 2 .

The AMB and multipropulsor compounds are single units that can be duplicated on a surface, in any pattern, using our new automated fabrication setup, as we now explore.

\subsection{Arrays of propulsors: active surface}

As a first test of active surfaces, we consider a triangular array of 55 AMBs in 10 lines, staggered so that no propulsor is being blocked by another one in front (see fig. 3a). A polydimethylsiloxane (PDMS) circular ring (2 $\mathrm{cm}$ inner diameter) is glued on the coverslip on which the AMBs are printed. It is filled with phosphate-buffered saline (PBS) and $2 \mu \mathrm{m}$ tracer beads before being closed with a PDMS disk. PBS was used since it increases the lifespan of the bubbles over that in pure water ${ }^{8}$. We spaced propulsors $100 \mu \mathrm{m}$ apart (from those adjacent on the same line and the closest in the line in front), thus producing an equilateral triangle array of side length $1 \mathrm{~mm}$. We can activate all the propulsors in the triangle simultaneously using the same ultrasound wave since its wavelength $\lambda$ is approximately 5 times the size of the triangle, as shown by the particle tracking velocimetry (PTV) analysis in fig. 3b (we used $2 \mu \mathrm{m}$ beads and FAST software ${ }^{10}$ for this).

We can see a net flow generated by this triangular array in fig. 3a. Each propulsor produces a pair of counter-rotating vortices similar to those seen on fig. 2a. However, there are unexpected non-uniformities in the flow. These are not due to the fabrication process but may originate from the tracer beads. Indeed we observed that the tracer beads tend to aggregate on the air/liquid interface around the opening, which affects the efficiency of the acoustic streaming and can almost cancel out the flow generation in some instances. Using smaller beads lead to no discernible differences. Another cause may be the non-uniformity of the acoustic field. Although the wavelength is significantly greater than the array size, there may be some modification of the acoustic field due to it crossing the PDMS. Therefore each AMB may not experience exactly the same acoustic excitation. This has been quantified on the histogram in fig. $3 c$ which shows the disparity of average velocities in front of the AMBs.

We are able to print $0.43 \mathrm{~mm}^{2}$ of propulsors, all containing bubbles that can be activated by the same acoustic wave. We now use these active surfaces, with AMBs placed closer than $100 \mu \mathrm{m}$, in order to generate collective flows for mixing inside microchannels.

\section{Active surface application: Microchannel mixing}

\subsection{Mixing at small scales}

Mixing in microchannels is known to be a challenging problem. At low Reynolds number, turbulence is not available and thus diffusion is solely due to molecular diffusion ${ }^{32}$. There has traditionally been two distinct types of micromixers: passive vs. active mixers, which exploit different strategies to generate mixing. Passive mixers use the shape of the microchannel ${ }^{38}$, either through carved grooves in the floor ${ }^{37}$, or through sophisticated shape designs ${ }^{20}$. In contrast, active mixers use external forces such as a magnetic field to activate a moving part inside the channel ${ }^{23}$.

Our active surfaces fall in the category of active mixers, since the acoustic field generates flow through the streaming of AMBs 8441 . The advantage of using individual micropropulsors, as we are now going to demonstrate, is the versatility of the arrangements able to mix. We now explore the effectiveness of different array shapes for mixing two fluid streams in a channel. One of our objectives is to use the minimum number of capsules for the maximum mixing efficiency.

\subsection{Setup}

We use an $80 \mu \mathrm{m}$ high microchannel of lateral width $e=1 \mathrm{~mm}$ (see fig. 4a-b), with two entrances: one is fed with a pure PBS solution while the other is fed with PBS+blue dye solution (see fig. 4a). The flow rate is controlled through two syringe pumps and was in the range $Q=2-4 \mu \mathrm{l} / \mathrm{min}$.

Experiments were performed with a $350 \mathrm{kHz}$ focused transducer (Olympus), in the acoustic pressure range of $P_{\mathrm{ac}}=100-2000 \mathrm{kPa}$. Pressure measurements are done using results from a separate experiment where a hydrophone (Onda) is placed behind a ring of PDMS of similar thickness to the microchannel in order to simulate the loss of pressure from transmitting sound from water to PDMS to PBS. The voltage applied to the transducer during the mixing experiments is then converted to the acoustic pressure evaluation deduced from the hydrophone measurements.

In order to assemble the microchannel with the glass coverslip on which the capsules are printed, marker lines are drawn on the glass on each side of the array of capsules. The microchannel is carefully placed on top after plasma treatment, with the pattern chosen from the possibilities of fig. $4 c$ in the middle of the microchannel. Note that the plasma treatment temporarily makes the surface of the propulsors hydrophilic so no bubbles are trapped. As such, a $12 \mathrm{hr}$ waiting period is thus required for the plasma treatment to wear off and before the microchannel is ready for use. In the case where the pattern is not perfectly positioned in the middle of the channel, we can move the demarcation line between the two liquids by adjusting the flow rate of one pump. The line of demarcation stabilises in a matter of seconds at the flow rates that we use.

\subsection{Protocol}

Experiments are conducted with cycles of 2-second ultrasound followed by two seconds at rest. Two seconds is sufficient to restore 
(a) PBS + blue dye

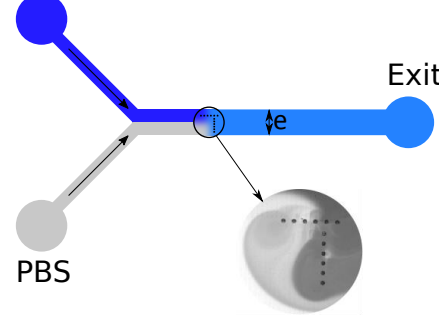

(b)

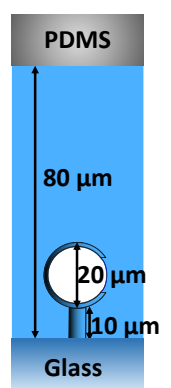

(c)

Line

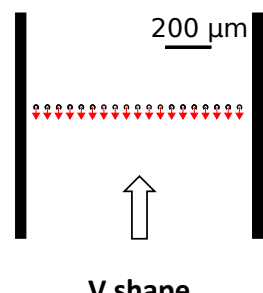

Tilted left/right
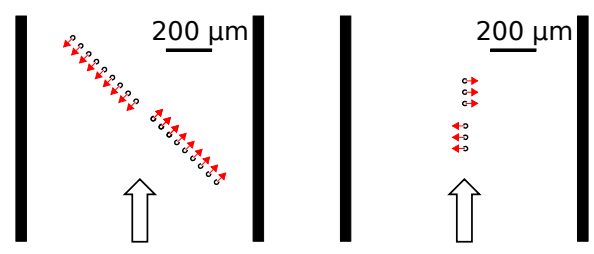

L shape
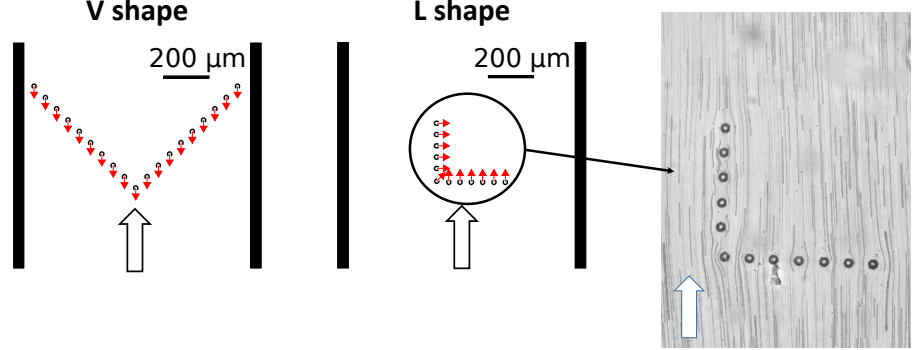

Fig. 4: (a) Y shaped microchannel used for mixing experiments. $e=1 \mathrm{~mm}$. (b) Scale of the capsules used compared to the height of the channel. (c) Arrangements of capsules tested for mixing. Red arrows: direction of the opening. White arrows: input flow direction. Blown up $L$ shape: experiment with a $1.2 \mathrm{~mm} / \mathrm{s}$ input flow and no ultrasound.

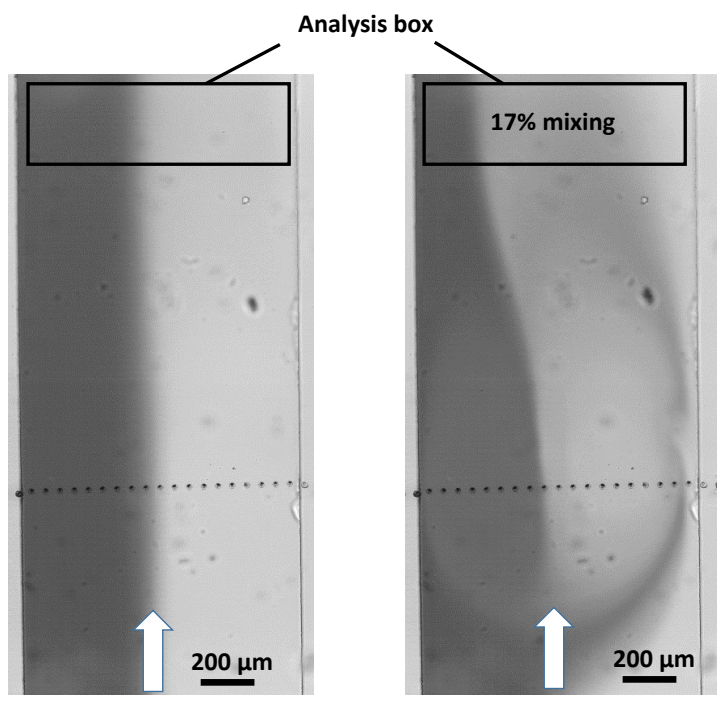

Fig. 5: Analysis of the mixing efficiency: example for a line shaped mixer. Left: no ultrasound. Right: with ultrasound. the flow to an unmixed state and prevent additive effects between two successive mixing cycles. It is also much greater than the mixing time that we will calculate below in section 5.1. The acoustic pressure is increased over the course of each experiment to investigate its effects on mixing and there are typically 20 to 50 cycles in each experiment.

In order to quantify mixing, we first define an 'analysis box' downstream of the pattern of AMBs (see fig. 5). The width of the box is equal to the width of the channel while its height has been set to 100 pixels $(287 \mu \mathrm{m})$. The distance from the last capsule to the beginning of the box is between 700 and $1100 \mu \mathrm{m}$. The box is always positioned sufficiently downstream so as not to include any of the vortices generated by the AMBs. We then measure the $R M I$ mixing efficiency (see details in ref. ${ }^{17}$ ) as $R M I=1-\frac{\sigma}{\sigma_{0}}$ where $\sigma=\sqrt{\frac{1}{N} \sum_{i=1}^{N}\left(I_{i}-\langle I\rangle\right)^{2}}$ and $\sigma_{0}=\sqrt{\frac{1}{N} \sum_{i=1}^{N}\left(I_{0 i}-\langle I\rangle\right)^{2}}$, and $N$ : number of pixels, $i$ : analysed pixel, $I_{i}$ : grey intensity of pixel $\mathrm{i},\langle I\rangle$ : average intensity of the box, $I_{0 i}$ : intensity of pixel $i$ in the unmixed state. A result with $R M I=0$ indicates no mixing while $R M I=100 \%$ means perfect mixing. Using the $R M I$ value allows us to compare experiments that may have different background lighting conditions (or different dyes) ${ }^{17}$ by normalising the standard deviation $\sigma$ by the standard deviation in the unmixed state $\sigma_{0}$.

\subsection{Micropropulsor array optimization}

AMB arrays are active mixers but their physical presence in the channel could also contribute to passive mixing. Separate experiments show that when the base flow passes near non-excited capsules it is deflected on a length scale close to the diameter of the capsules before resuming its linear trajectory (see fig. 4c). Passive 
mixing is thus negligible so we will attribute mixing effectiveness in what follows to the flows generated by the active capsules.

\subsubsection{Line mixers: effect of orientation}

In order to make the microchannel assembly process easier, we first designed an active surface consisting of a straight line of propulsors, each $50 \mu \mathrm{m}$ apart (centre to centre), stretching across the channel and pointing against the input flow. This is easy to assemble since a line of propulsors longer than the channel width can be printed, and thus the centre of the array does not need to be aligned with the centre of the channel when attaching the microchannel and substrate, unlike with other patterns. The line has 19 AMBs within the channel, each containing an $18 \mu \mathrm{m}$ diameter bubble.

The flow generated is shown in fig. $6 \mathrm{a}$ and takes the form of a large vortex pair, taking up the whole width of the channel, with each vortex $\sim 500 \mu \mathrm{m}$ wide. This is a large collective flow generated by individual small elements. Mixing-wise, it only reaches $R M I=61 \%$ (see fig. 6b) and the efficiency decreases for higher acoustic pressures. The effect is clearer when the peak mixing efficiency measured during each pressure cycle is plotted against the acoustic pressure (see fig. 6c). This decrease in efficiency is due to the capsules gradually filling with water. Our observations suggest therefore that when submitted to the permanent flow in the microchannel and forced with ultrasound, bubbles tend to disappear much quicker than when placed in a liquid bulk. As a comparison, the propulsors used in the triangle experiments of subsection 3.2 can be used for several hours. Note that in the triangle experiments, silanisation even further increases the bubbles' lifespan as it makes the capsules hydrophobic ${ }^{34}$ but silanisation is not an option in microchannels where a silanised surface leads to poor wetting and the formation of very large bubbles. Nevertheless, this experiment does confirm that closely-placed AMBs in a microchannel can generate large flows.

Next we tilt the line shape by $45^{\circ}$ and point half of the AMBs down/left and half up/right (see fig. 7a). The aim was to both lengthen the lifespan of the bubbles by having them not pointing directly into the permanent flow, and to increase the mixing efficiency. Both goals were achieved; indeed, for 150 seconds almost all propulsors kept air inside themselves and a mixing efficiency of 90\% (fig. 7b) was reached. However this shape requires a large number of capsules in order to fill the channel and to achieve good mixing.

This then motivated a study of a left/right line consisting of only 6 propulsors (see fig. 8a), three times fewer than the tilted left/right mixer. All propulsors there stay active for the whole 250 seconds of the experiment and reach a maximum mixing efficiency of $98 \%$ (fig. 8c). Therefore we are able to fully mix a wide channel ( $e=1 \mathrm{~mm}$ ) with only 6 propulsors, arranged in a shape that takes as little as 90 minutes to print. We propose that the high efficiency of this pattern is due to the two vortices communicating with each other as seen in fig. 8a, exchanging fluid from the two sides of the channel.

In order to investigate whether we can predict this behaviour numerically we used the Stokeslet model described in subsection 2.2. The numerical flow field shown in fig. $8 \mathrm{~b}$ contains similar (a)

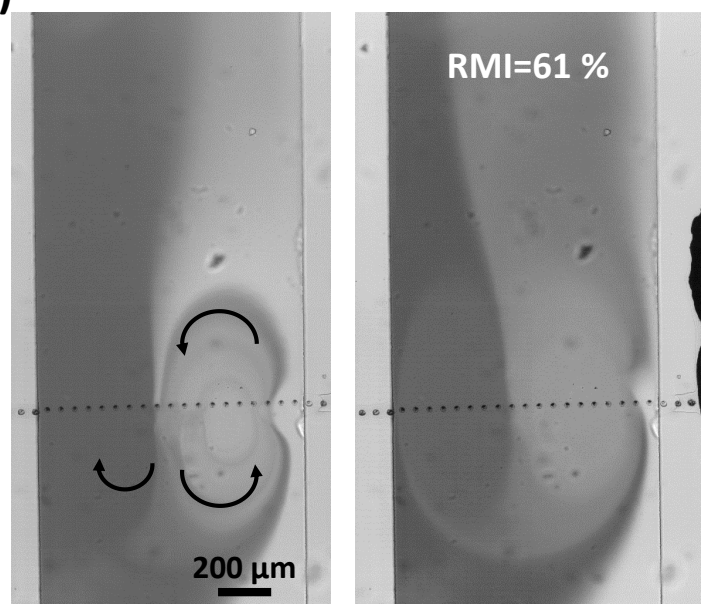

(b)
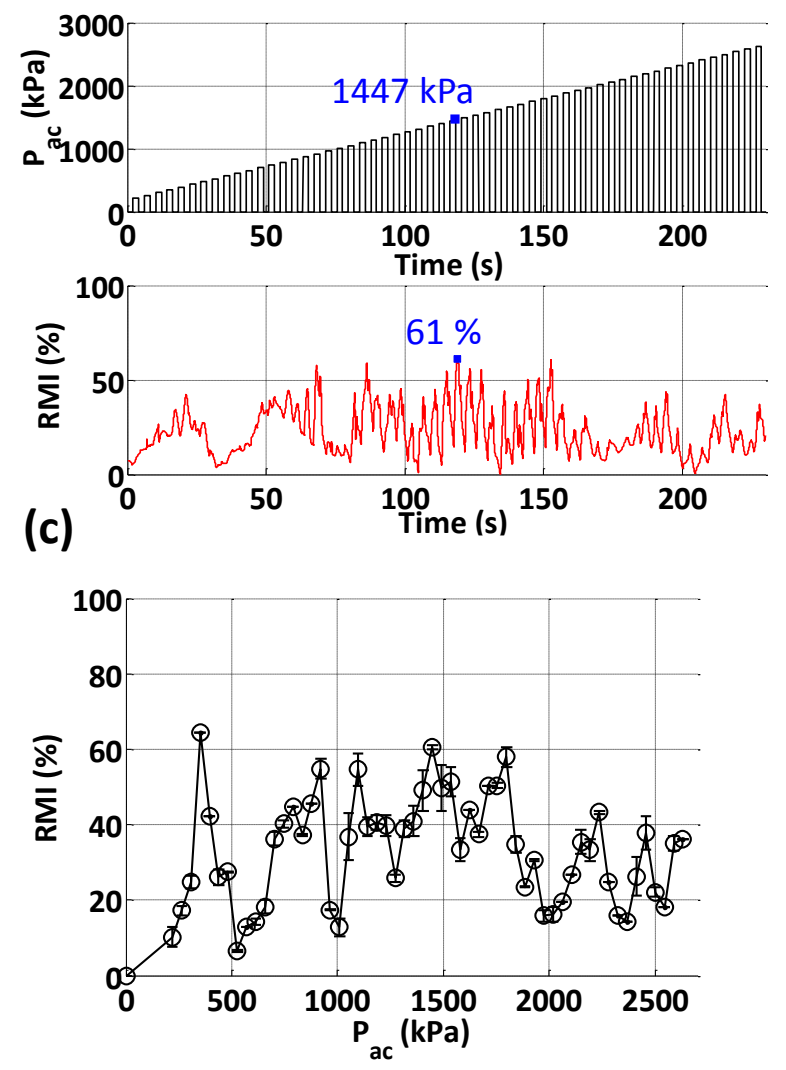

Fig. 6: Line shape: (a) Left: Vortices at the start of the $P_{\mathrm{ac}}=1.45 \mathrm{MPa}$ mixing cycle, and right: maximum mixing during the cycle; (b) Acoustic pressure cycles and corresponding mixing efficiency; (c) Peak mixing efficiency during each pressure cycle against acoustic pressure. 
(a)
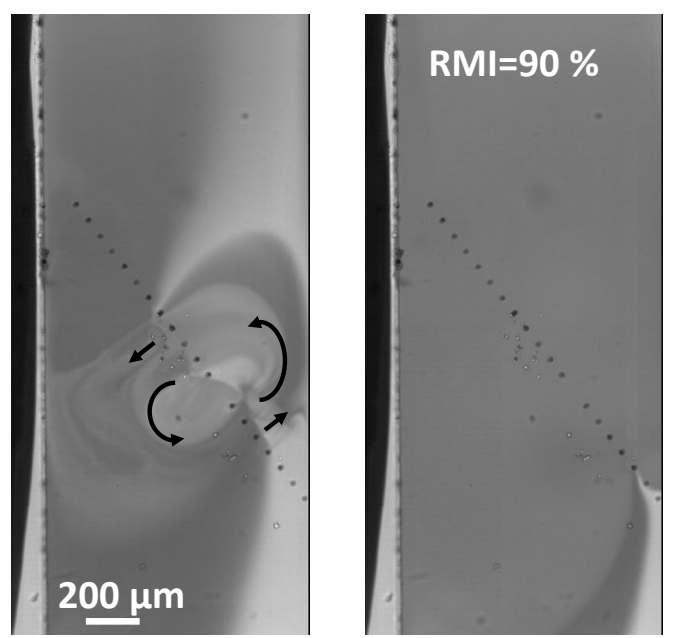

(b)

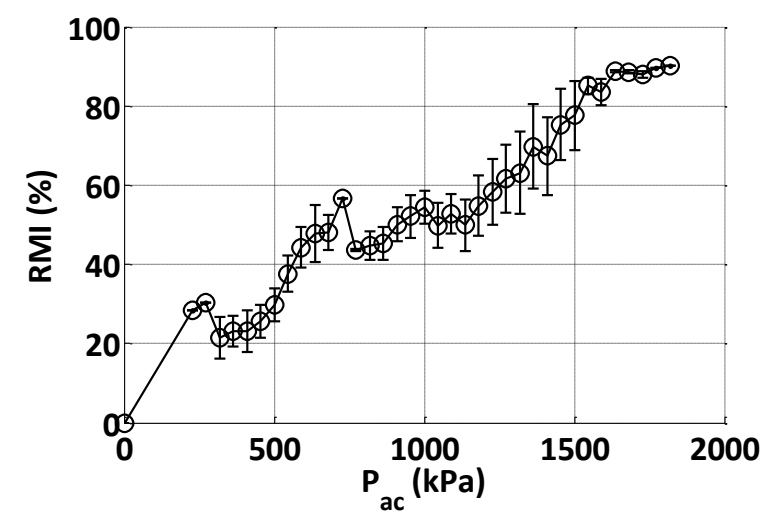

Fig. 7: (a) Tilted left/right shape, $P_{\mathrm{ac}}=1.82 \mathrm{MPa}$; (b) Mixing efficiency against acoustic pressure. (a)

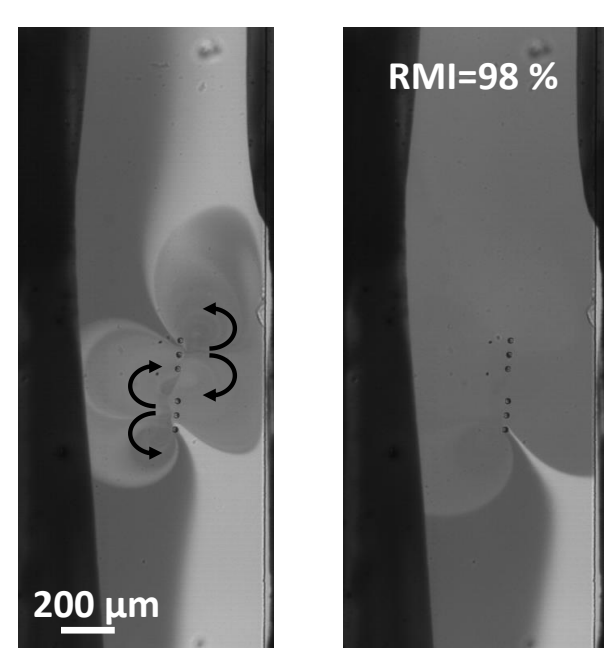

(b)

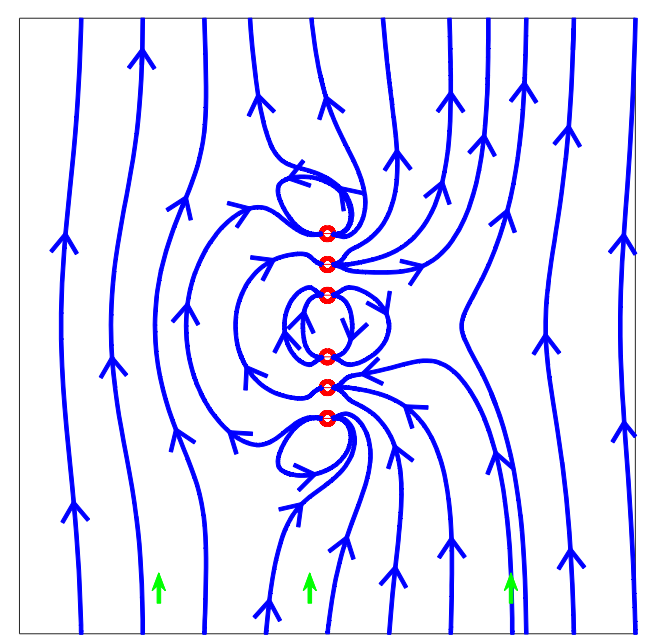

(c)

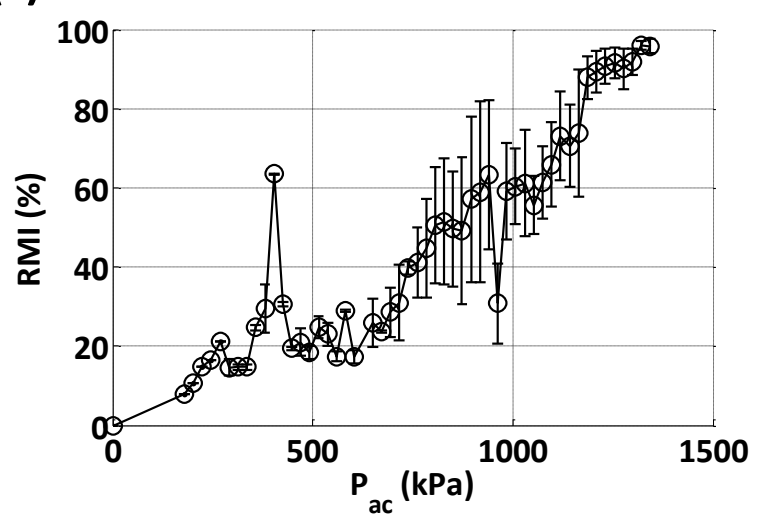

Fig. 8: (a) Left/right shape, $P_{\mathrm{ac}}=1.34 \mathrm{MPa}$. The black shadows on the left and right are due to the markers we use for precisely positioning the mixers in the middle of the channel; (b) Streamline prediction with $1.2 \mathrm{~mm} / \mathrm{s}$ background flow, a force per Stokeslet $f_{\mathrm{st}}=9 \mathrm{nN}$ hence a maximum velocity of $v_{\text {th }}=78 \mathrm{~mm} / \mathrm{s}$; (c) Mixing efficiency against acoustic pressure. 
(a)

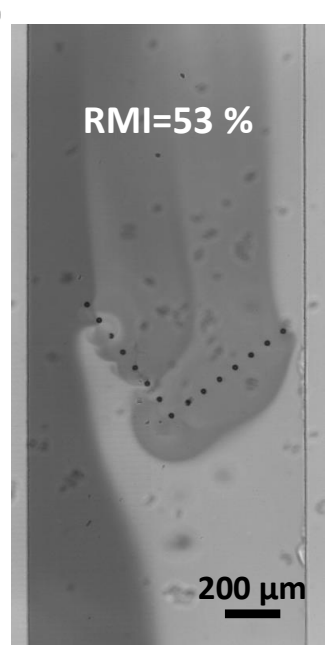

(b)



(c)



Fig. 9: $V$ shape mixer. (a) Low acoustic pressure, $P_{\mathrm{ac}}=224 \mathrm{kPa}$, propulsors full of air (b) $P_{\mathrm{ac}}=761 \mathrm{kPa}$, the propulsors on the right have lost their air, and mixing only occurs on the left side of the channel (c) $P_{\mathrm{ac}}=1343 \mathrm{kPa}$, all capsules are full of water.

vortex patterns to the experiments for a base flow velocity of 1.2 $\mathrm{mm} / \mathrm{s}$ and quantitatively similar parameters as the experiments. More importantly, it predicts the exchange of liquid from one side of the channel to the other.

\subsection{2 $\quad V$ shape mixers}

Motivated by trying to separate the flow in order to maximise mixing, we developed a $V$-shaped-mixer (see fig. 9), aligning the point in the $V$ shape with the line of demarcation between the two liquids. We examined the effect of changing the distance between propulsors on the mixing efficiency. Gaps of $100 \mu \mathrm{m}$ and above between propulsors gave insignificant mixing (RMI $<20 \%$ ). Smaller gaps of $50 \mu \mathrm{m}$ performed far better but, similarly to the line mixers, the lifespan of the bubbles is greatly diminished when propulsors are facing into the flow. In the case of the $V$ mixer, the bubbles can disappear in 7 or 8 mixing cycles, as seen in fig. $9 \mathrm{~b}$. This explains why the maximum mixing is reached (for $50 \mu \mathrm{m}$ gaps) at $P_{\mathrm{ac}}=806 \mathrm{kPa}$ and decreases at higher pressures (fig. 9c). However, this pattern is the most efficient at very low acoustic pressure, where it reaches $\mathrm{RMI}=59 \%$ for $P_{\mathrm{ac}}=224 \mathrm{kPa}$. (a)

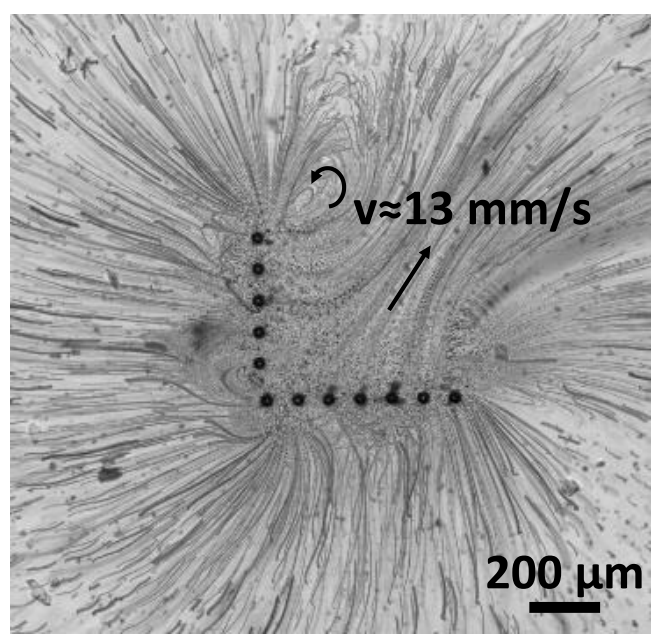

(b)

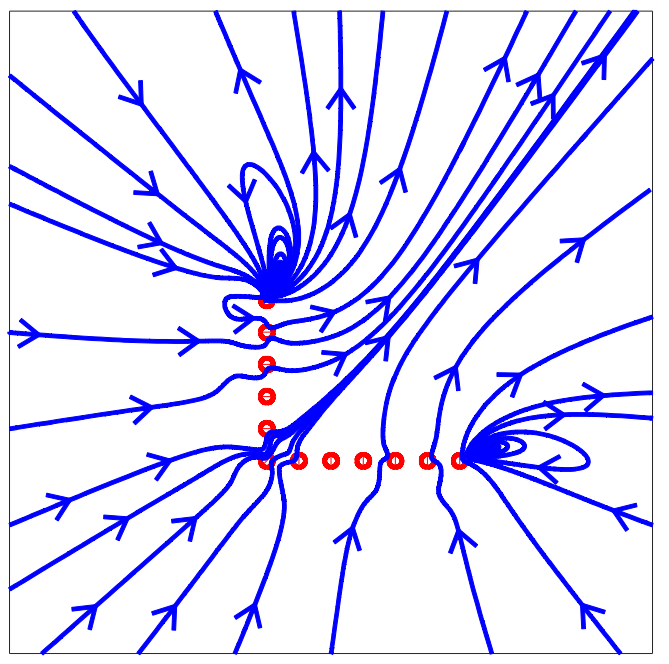

Fig. 10: $L$-shape mixer in an open pool: (a) $P_{\mathrm{ac}}=351 \mathrm{kPa}$, no large vortex formed; (b) Model prediction for $f_{\mathrm{st}}=4.5 \mathrm{nN}$ corresponding to a maximum velocity of $v_{\text {th }}=39 \mathrm{~mm} / \mathrm{s}$.

\subsection{3 $L$ shaped mixers: formation of a vortex pair and mixing time}

We next designed a $L$ mixer, inspired by the natural phenomenon of fire whirls which forms when trees in the shape of an $L$ burn inducing a wind vortex which fire is channelled into ${ }^{22}$. The $L$ mixer was designed to break the symmetry of the flow and generate a large mixing vortex. We now look in detail at the formation of this mixing vortex.

Firstly, we place the $L$ propulsor arrangement in an open pool and investigates if a mixing vortex is generated. We use a circular pool without a ceiling, made in a PDMS cell filled with PBS. Unlike with the triangle arrangement of section 3.2 , this active surface (fig. 10a) induces a collective flow whose shape agrees with our numerical model (fig. 10b). A collective flow is induced by the $L$ and not the triangular arrangement because of the smaller distance 
(a)



(d)

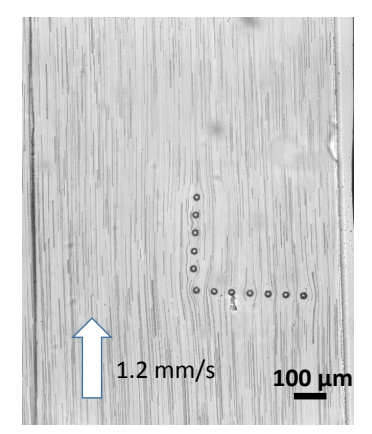

(b)

(e)



(c)



(f)



Fig. 11: (a-b) Vortex formed in a $80 \mu \mathrm{m}$ high microchannel, $5 \mathrm{~mm}$ circular pool; (a) $P_{\mathrm{ac}}=1.53 \mathrm{MPa}$; (b) Prediction of the simulations with a ceiling added for $f_{\mathrm{st}}=4.5 \mathrm{nN}$ and $v_{\mathrm{th}}=39 \mathrm{~mm} / \mathrm{s}$. Dashed black line: position and width of the vertical cut on (c); (c) Vertical cut view of the streamlines for the one wall case (no ceiling) and two wall case (with ceiling); (d-f) Formation of the vortex in a $80 \mu \mathrm{m}$ high microchannel, $1 \mathrm{~mm}$ wide, with increasing acoustic pressure; (d) $P_{\mathrm{ac}}=0$; (e) $P_{\mathrm{ac}}=93 \mathrm{kPa}$; (f) $P_{\mathrm{ac}}=216 \mathrm{kPa}$.

between propulsors (50 $\mu \mathrm{m}$ for the $L$ vs. $100 \mu \mathrm{m}$ for the triangle). However in this open pool we do not observe the formation of a large vortex but only small individual vortices generated at both extremities of the $L$ (both experimentally and numerically).

We next confine the $L$ in a $80 \mu \mathrm{m}$ high microchannel in order to investigate the role played by the top wall. The channel has the shape of a circular pool of diameter $5 \mathrm{~mm}$. The specific microchannel used for fig.11a-b had a thick PDMS layer, so attenuates ultrasound from the transducer more than usual since higher acoustic pressures were used for these experiments than the ones shown in fig. 10. However the streaming velocities generated by the propulsors $(v=10-30 \mathrm{~mm} / \mathrm{s})$ were comparable.

In this confined channel, a large leftward vortex is observed, together with a rightward vortex (see fig. 11a), in agreement with numerical simulations (fig. 11b). From this result, we deduce that the non-constrained flow in fig. 10 is three-dimensional, flowing upward, and that the addition of a ceiling constraining that upward flow is compensated for by the formation of a pair of vortices. This hypothesis is confirmed by simulations. A vertical cut alongside the length of the $L$ shows that with no ceiling, the liquid flows upward. The ceiling blocks that flow (see fig. 11c) and conservation of mass must result in the formation of two vortices.

We next confine the $L$ further, placing it in an $e=1 \mathrm{~mm}$ wide and $80 \mu \mathrm{m}$ high microchannel. We will analyse the formation of its large vortex as $P_{\mathrm{ac}}$ is increased, see fig. 11d-f. At lower pressures, we see in fig 11e individual vortices, including the backward flows characteristic of individual propulsors on $10 \mu \mathrm{m}$ poles (see fig. 2a). As the pressure increases, the backward flow becomes small compared to the strong forward flow, and a large leftward vortex takes shape (see fig. 11f). There is a threshold pressure at which the vortices form (specifically, $P_{\mathrm{ac}}=216 \mathrm{kPa}$ ).

Considering the flow velocities in these vortices in more detail, we again use the $L$ in a $80 \mu \mathrm{m}$ high circular pool, the setup which produced fig. 11a-b. Particles alongside the vortices follow nearellipsoidal trajectories, but their average velocities change as they move around the vortex. The vorticity analysis in fig. 12a (using the freeware FAST ${ }^{10}$ ) confirms that it is a rotational vortex. The mean velocities of the particles is shown in fig. $12 \mathrm{~b}$. The particles reach a maximum of $27 \mathrm{~mm} / \mathrm{s}$ inside the $L$ and decrease as they follow the elliptical trajectory further away from the $L$. One of the leftward trajectories is analysed in detail in fig.12c. Starting inside the $L$ the velocity is at a maximum. The velocity along the vortex $v_{t}$ then decreases until the attracting force from the AMBs affect the particles again and the velocity increases. There is thus no simple relationship between the particle velocity and the radial distance from the centre of the vortices. From this analysis, we can also calculate the time $\tau_{\mathrm{m}}$ taken for particles to complete a full loop of the vortex. This is shown on the insert of fig. 12c, which reveals that it takes between 5 and $400 \mathrm{~ms}$ to complete a loop of the vortex, depending on the distance from the vortex centre.

We finally test the mixing efficiency of the $L$ pattern inside our $Y$-shaped microchannel. Our measurements show that the $L$ shape 
(a)



(c)

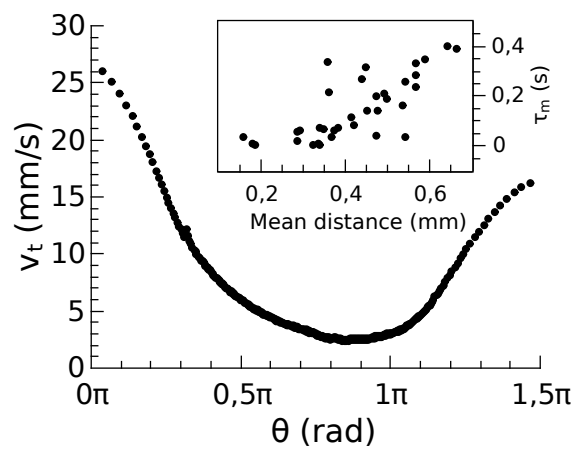

Fig. 12: (a) Vorticity analysis; (b) Mean velocity of the particles and notations; (c) Particle velocity $v_{t}$ against $\theta$ for a complete loop. Insert: travel time $\tau_{m}$ against mean radial distance from the centre of the vortex. (a)

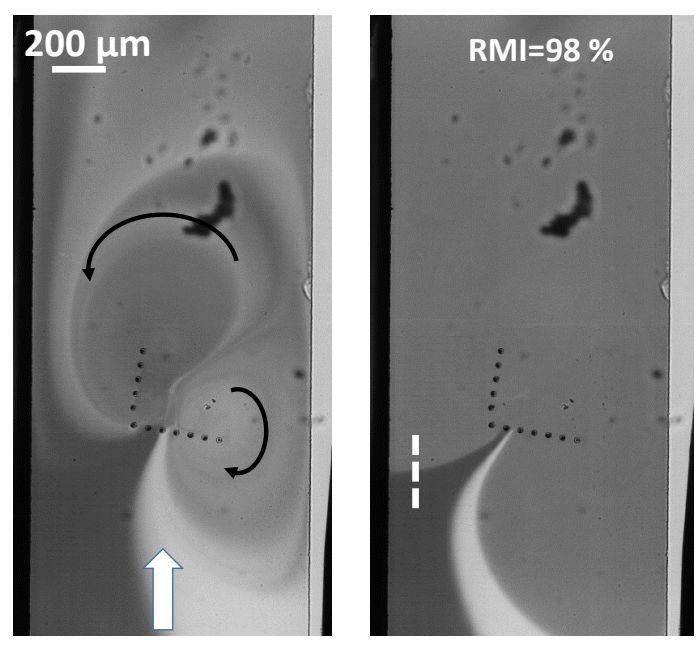

(b)

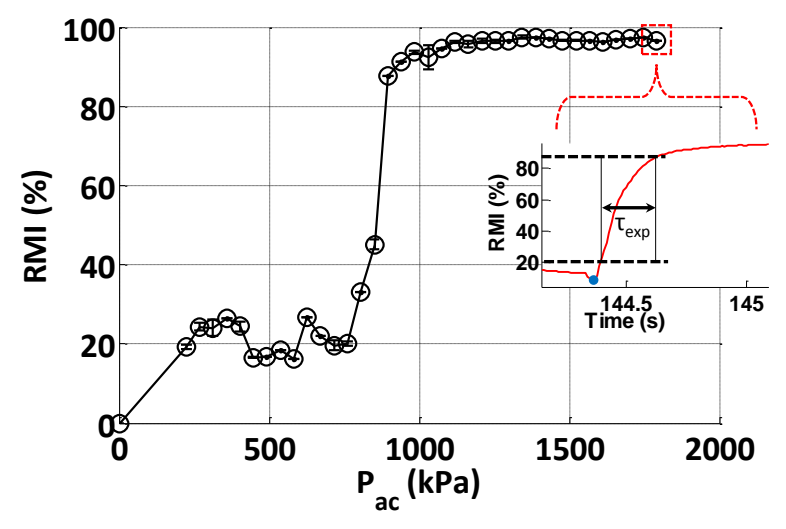

Fig. 13: (a) $L$ shape in an $e=1 \mathrm{~mm}$ microchannel, $P_{\mathrm{ac}}=1.34 \mathrm{MPa}$ (dashed white line: segment taken to calculate the mixing time with ref. ${ }^{4}$ ); (b) Mixing efficiency against acoustic pressure. Insert: RMI against total experiment time, with $\tau_{\text {exp }}$ the time it takes to go from $R M I=20 \%$ to $R M I=90$ $\%$. The blue circle represents the moment the ultrasound is turned back on. 
gives highly efficient and sustained mixing as shown in fig. 13 . The generated vortices produce nearly perfect mixing in our microchannel for $P_{\mathrm{ac}} \geq 1 \mathrm{MPa}$ (see fig. 13b), which is a significantly lower pressure threshold value for nearly perfect mixing than the other array shapes. This arrangement is the most efficient of all the patterns we considered. Moreover, the propulsors suffer significantly less from loss of air than any other patterns since they do not face into the permanent flow. This pattern is also robust, since experiments showed the level of mixing is unaffected whether the $L$ is placed in the centre of the channel or slightly closer to either side wall, or even if the $L$ is tilted. This makes the $L$ array an excellent candidate for microfluidic applications.

\section{Discussion}

\subsection{Mixing process and mixing time}

Microfluidic mixers aim, essentially, to accelerate molecular diffusion. One method, used by grooved microchannels, is to generate chaotic mixing by folding the flow onto itself. Another method, exploited by our AMB arrays, becomes apparent when a blob of dye is placed inside a thin channel and a laminar flow is imposed. The dye is stretched, taking on the parabolic shape of the flow. At the front of the parabola, the dye diffuses towards the walls, while at the back of the parabola it diffuses inward towards the channel centre. This classical process is called Taylor-Aris dispersion 6,39. Stretching the liquid with high shear rates can increase mixing efficiency as used by circular mixers ${ }^{33}$.

The Péclet number for our experiments is given by $P \mathrm{e}=\frac{h v}{D}$ where $v=1-100 \mathrm{~mm} / \mathrm{s}$ is the flow velocity generated by the propulsors, $h=80 \mu \mathrm{m}$, and $D \approx 10^{-5} \mathrm{~cm}^{2} / \mathrm{s}$ so that $P \mathrm{e} \approx 10^{2}-10^{4}$. Due to the very strong flows generated by our propulsors, the Péclet number of our experiments is 1 to 2 orders of magnitude larger than is common in microfluidic experiments. This confirms that the convection-diffusion at play in Taylor-Aris dispersion will be significant.

Assuming a steady incompressible pressure-driven laminar flow in the channel, the dispersion is governed by the equation:

$$
\frac{\partial}{\partial t} c+v \cdot \nabla c=D \nabla^{2} c
$$

where $c$ is the concentration and $v$ the velocity field ${ }^{33}$. For long time scales and a narrow channel cross-section $(h \ll e)$, the effective diffusion coefficient $D_{\text {eff }}$ is $D_{\text {eff }}=D\left(1+\kappa P e^{2}\right)$, where $\kappa$ is a shape coefficient. With $P e \gg 1$, the relative significance of Taylor dispersion to molecular diffusion is $\kappa P \mathrm{e}^{214}$. If we assume $v \approx 20$ $\mathrm{mm} / \mathrm{s}$ such as in fig. $10 \mathrm{a}$ and $11 \mathrm{a}$, then $\frac{D_{\text {eff }}}{D} \approx 2.610^{6} \kappa$. This requires an approximate value for $\kappa$ which, in general, is difficult to compute exactly. But we can estimate $\kappa$ as being between its lower limit of two infinite parallel plates $(\kappa=0.0048)$ and its higher limit of a trapezoidal channel $(\kappa=0.0329)^{14}$. Thus, in our case, Taylor dispersion contributes at least $99.99 \%$ to the total dispersion. In addition, we note that the Taylor-Aris diffusion time scale $\tau_{\text {Taylor }}$ is $\tau_{\text {Taylor }}=\frac{e^{2}}{D_{\text {eff }}}=10-80 \mathrm{~ms}^{33}$.

Mixing time is typically defined as the time taken for a particle to travel from an unmixed to a perfectly mixed region ${ }^{4}$. However, for our mixers, the border between the unmixed and mixed regions is dynamic, making it difficult to exploit this definition to measure the mixing time. This is corroborated when analysing the grey profile alongside the segment defined by the dashed white line on fig 13a as using this definition leads to an unrealistic mixing time of $10 \mathrm{~ms}$.

For the $L$-shaped mixer we instead hypothesise that the mixing is of the same order of magnitude as $\tau_{\mathrm{m}}$, the time taken for a fluid particle or tracer to complete a loop inside the vortex. For $P_{\mathrm{ac}}=1.53 \mathrm{MPa}$, a bead takes $\tau_{\mathrm{m}}=10-400 \mathrm{~ms}$ to perform a complete loop of a vortex (fig. 12c), depending on its distance from the centre of the vortex. Therefore, a good approximation for the mixing time in our $e=1 \mathrm{~mm}$ microchannel, is to average $\tau_{\mathrm{m}}$ over all tracers within $500 \mu \mathrm{m}$ of the vortex centre (assuming the vertical branch of the $L$ is in the middle of the channel). This gives a value of $\tau_{\text {average }}=111 \mathrm{~ms}$, which is much lower than the 2 -second cycle period we use in our experiments. Unsurprisingly, $\tau_{\text {average }}$ is also on the same order of magnitude as the Taylor diffusion time scale $\tau_{\text {Taylor }}$. The mixing time will be on the same order of magnitude as $\tau_{\text {average, }}$ since we expect mixing will take only a few loops. On the insert in fig. 13b the RMI measurements are plotted against time, focusing on the start of a new cycle when the driving force is turned on. The acoustic pressure was $P_{\mathrm{ac}}=1.48 \mathrm{MPa}$, similar to the experiment of fig. 11a. The results show a steep increase in RMI before plateauing. Here the velocity inside the vortex is much greater than the input flow and the mixed fluid reaches the analysis box in only $30 \mathrm{~ms}$ as compared to at low acoustic pressure where it takes about $0.3 \mathrm{~s}$ for the mixed liquid to be advected to the analysis box downstream (see fig. 5). To go from RMI=20\% to $\mathrm{RMI}=90 \%$, it takes $\tau_{\exp }=380 \mathrm{~ms}$ so just under 4 loops.

\subsection{Theoretical outlook}

Using the experimental mixers as templates we finally considered variations on their design and addressed theoretically their impact on mixing using the method described in sec. 2.3. Specifically, we considered rotations of both the mixers and of their rows of AMBs (or the AMBs themselves). We also varied the starting position of the fluid boundary across the width of the channel, considering the line extension at every $10 \mu \mathrm{m}$ position across a $1 \mathrm{~mm}$ width.

Our theoretical results indicate that the position of the boundary between the two fluids to be mixed relative to the AMBs does affect the mixing efficiency. If there is a circulation in the flow, it will benefit mixing if it is able to pull in both fluids rather than just rotating one of them. The position of the boundary affects which local features stretch and contract and by how much (see fig. 14), although some mixers (like the long left/right mixer) do have wide bands of similar boundary stretching. The two biggest differences in behaviour is that either the boundary is stretched but passes out the other side of the mixer or it is pulled and caught in local vortices.

Our analysis best compares the cases where the boundary passes completely through the mixer, which is useful for a mixer which is permanently 'on' as vortices trap fluid, delaying fluid from progressing down the channel even once it is mixed. In this scenario the best of the experimental mixers was a long left/right followed closely by the tilted left/right mixers and then the $L$ shape (see 




(b)
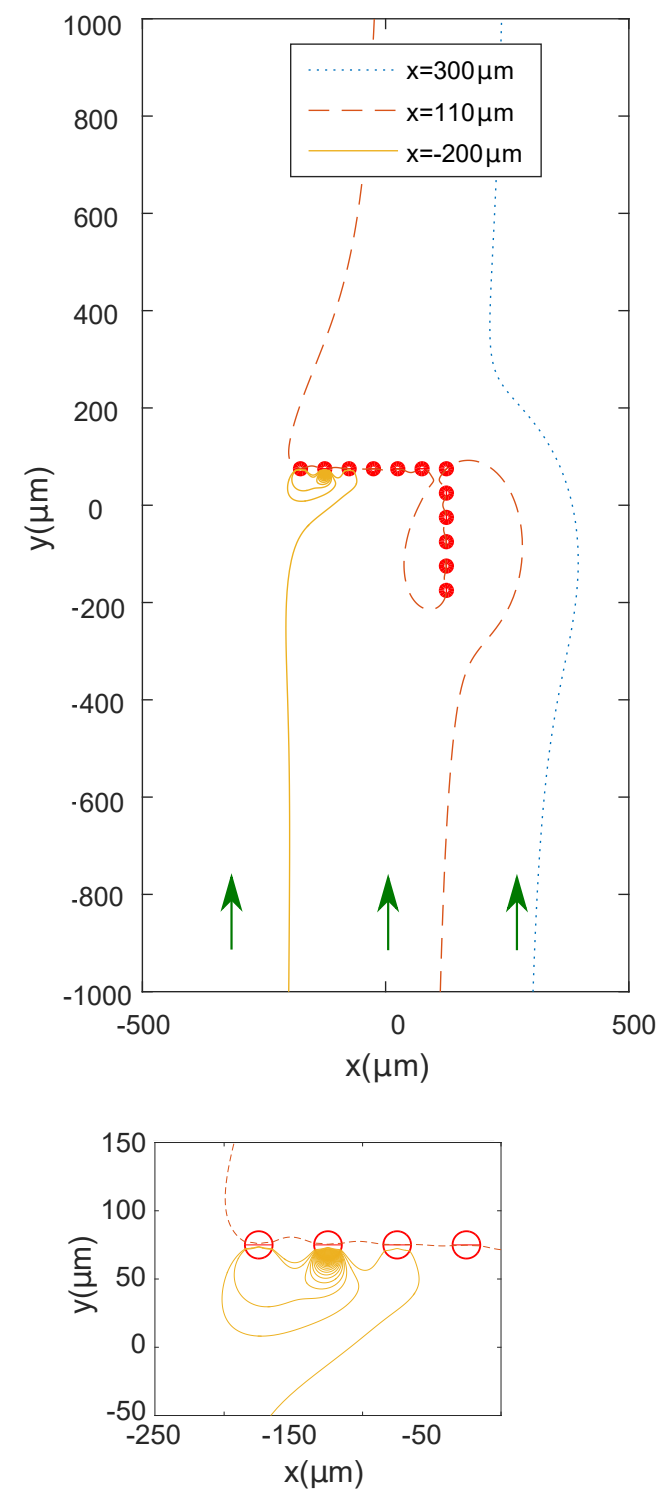

Fig. 14: (a) Modified L shape; (b) Shape of the boundary as it passes through the $L$ mixer on (a) for three boundary starting positions. The insert zooms in on the local vortex. (a)

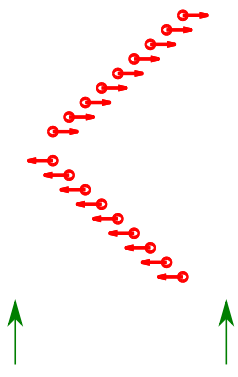

(b)

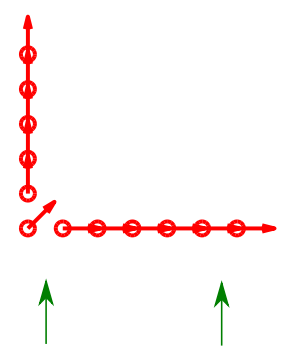

Fig. 15: Two variations of the (a) left/right and (b) $L$ shape experimental mixers which produce good continuous mixing. The black line indicates initial position of the two fluid boundary. The green arrow indicates the direction of the base flow

fig. 4). In addition the left/right mixer has a wide band of over $300 \mu \mathrm{m}$ where a similar high level of stretching is observed, very useful when diffusion starts to smudges the boundary. However, theoretically better mixing can be achieved with mixers such as those shown in fig. 15 . For the $L$-mixer, generally good mixing is observed theoretically when the two rows of AMBs point along their own length.

We expect vortices to create the best mixing, particularly when the mixer is turned 'on' and 'off' as in the experiments. Local vortices cause the greatest stretching, but the boundary then became tightly wrapped so the diffusion length scale becomes very significant. If the AMB strength was increased, corresponding to higher driving pressures, vortices would become larger in size thus potentially reducing this issue. However the unconstrained large vortices throw flow out sideways rather than capturing it thus the side walls are significant. Future work could include adding side walls to our model and consider stretching of the boundary already within the mixer.

Both mixing arangements of AMBs presented in fig. 15 were studied experimentally. The modified L shape suffered from an unexpected issue of short bubble lifespan in the horizontal line of AMBs pointing across the channel; it was only able to reach $\mathrm{RMI}=37 \%$ for $P_{\mathrm{ac}}=448 \mathrm{kPa}$ before the bubbles disappeared (not shown). This new $L$ shape was already rotated $180^{\circ}$ from fig. 4(a) to fig. 15 so the AMBs along the vertical line of the L would not point against the flow, but we did not anticipate the AMBs on the horizontal line would experience issues as well. Clearly these lifespan issues would not be identified by the numerical model. The variation of the left/right mixer was far more promising experimentally, with $\mathrm{RMI}=65.5 \%$ obtained at a low acoustic pressure of $P_{\mathrm{ac}}=537 \mathrm{kPa}$ (see fig. 16b). However at higher pressures the AMBs pointing to the right lost their bubbles. Future experimental work should tackle the issue of bubble lifespan, for example by making the printed capsules hydrophobic.

\section{Conclusion}

In this paper we have demonstrated that, due to the versatility of our fabrication setup, bubble-based acoustic micropropulsors can be arranged in any pattern and so generate a rich variety of flows. Those flows are sustained, powerful, and well predicted by our hydrodynamic model. Moreover, a multitude of objects can be activated by the same acoustic wave. Furthermore, these 
(a)
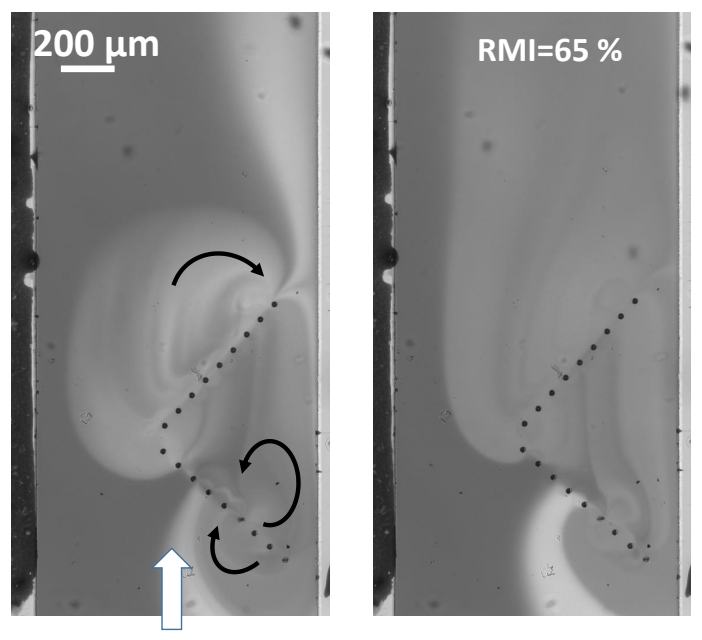

(b)

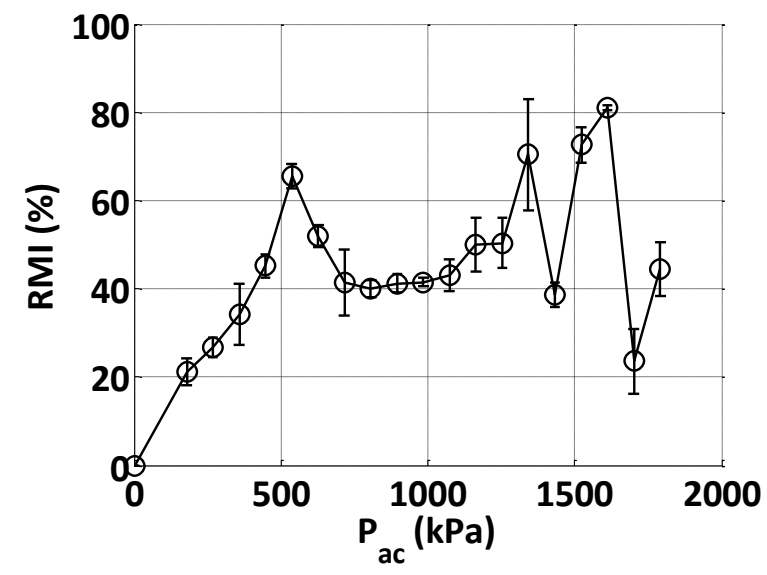

Fig. 16: (a) Modified left/right shape, $P_{\mathrm{ac}}=537 \mathrm{kPa}$; (b) Mixing efficiency against acoustic pressure. arrays have shown great promise for use as mixers in microchannels. A pattern of individual micropropulsors can generate large vortices close to the millimetre scale. Those vortices, present for shallow microchannels, mix through Taylor-Aris dispersion in very short time-scales. They can be predicted by modelling the propulsors as individual flow singularities bound by two parallel walls. Our experiments demonstrate the possibility of using relatively few propulsors for mixing a $1 \mathrm{~mm}$ wide microchannel: an $L$ pattern containing 12 propulsors is the most efficient from a relatively low acoustic pressure, with long lifespan bubbles, and a left/right pattern with only 6 propulsors is able to fully mix at slightly higher pressures.

\section{Acknowledgments}

P. M. acknowledges financial support from the European Community's Seventh Framework Programme (FP7/2007-2013) ERC Grant Agreement Bubbleboost no. 614655. This work has been performed with the help of the "Plateforme Technologique Amont" de Grenoble, with the financial support of the "Nanosciences aux limites de la Nanoélectronique" foundation. This work was partially funded through a Marie Curie CIG grant (EL) and through EPSRC (TS). Thanks also to M.-C. Jullien for fruitful discussion on Taylor dispersion, and to Microlight ${ }^{1}$ for writing the Autofocus script.

\section{References}

1

2

3 D. Ahmed, M. Lu, A. Nourhani, P. E. Lammert, Z. Stratton, H. S. Muddana, H. Crespi, V, and T. J. Huang. Selectively manipulable acoustic-powered microswimmers. Sci. Rep., 5:9744, 2015.

4 Daniel Ahmed, Xiaole Mao, Jinjie Shi, Bala Krishna Juluri, and Tony Jun Huang. A millisecond micromixer via single-bubblebased acoustic streaming. Lab Chip, 9(18):2738-41, September 2009.

5 J. Ainley, S. Durken, R. Embid, P. Boindala, and R. Cortez. The method of images for regularized stokeslets. J. Comput. Phys., 227(9):4600-4616, 2008.

6 R. Aris. On the dispersion of a solute in a fluid flowing through a tube. P. Roy. Soc. Lond. A. Mat., 235(1200):67-77, 1956.

7 Anthony K. Au, Nirveek Bhattacharjee, Lisa F. Horowitz, Tim C. Chang, and Albert Folch. 3d-printed microfluidic automation. Lab Chip, 15:1934-1941, 2015.

8 Nicolas Bertin, Tamsin A. Spelman, Olivier Stephan, Laetitia Gredy, Michel Bouriau, Eric Lauga, and Philippe Marmottant. Propulsion of bubble-based acoustic microswimmers. Phys. Rev. Applied, 4:064012, Dec 2015.

9 J. R. Blake. A note on the image system for a stokeslet in a noslip boundary. Proc. Camb. Phil. Soc., 70(2):303-310, 1971. 
10 Thomas Combriat.

11 Kevin Conlisk and Gerard M. O'Connor. Analysis of passive microfluidic mixers incorporating $2 \mathrm{~d}$ and $3 \mathrm{~d}$ baffle geometries fabricated using an excimer laser. Microfluid. Nanofluid., 12(6):941-951, 2012.

12 R. Cortez and D. Varela. A general system of images for regularized stokeslets and other elements near a plane wall. $J$. Comput. Phys., 285:41-54, 2015.

13 Ricardo Cortez. The method of regularized stokeslets. SIAM J. Sci. Comput., 23(4):1204-1225, 2001.

14 Subhra Datta and Sandip Ghosal. Characterizing dispersion in microfluidic channels. Lab Chip, 9:2537-2550, 2009.

15 Jian Feng, Junqi Yuan, and Sung Kwon Cho. Micropropulsion by an acoustic bubble for navigating microfluidic spaces. Lab Chip, 15:1554-1562, 2015.

16 Hua Gong, Adam T. Woolley, and Gregory P. Nordin. High density $3 \mathrm{~d}$ printed microfluidic valves, pumps, and multiplexers. Lab Chip, 16:2450-2458, 2016.

17 Ali Hashmi and Jie Xu. On the quantification of mixing in microfluidics. J. Lab. Autom., 19(5):488-491, 2014.

18 Sascha Hilgenfeldt, Detlef Lohse, and Michael Zomack. Sound scattering and localized heat deposition of pulse driven microbubbles. J. Acoust. Soc. Am., 107(6):3530-3539, 2000.

19 Rustem F. Ismagilov, Abraham D. Stroock, Paul J. A. Kenis, George Whitesides, and Howard A. Stone. Experimental and theoretical scaling laws for transverse diffusive broadening in two-phase laminar flows in microchannels. Appl. Phys. Lett., 76(17):2376-2378, 2000.

20 F. Jiang, K. S. Drese, S. Hardt, M. Kupper, and F. Schonfeld. Helical flows and chaotic mixing in curved micro channels. AIChE J., 50(9):2297-2305, 2004.

21 Andrew Evan Kamholz and Paul Yager. Molecular diffusive scaling laws in pressure-driven microfluidic channels: deviation from one-dimensional einstein approximations. Sensor Actuat. B Chem, 82(1):117 - 121, 2002.

22 Kazunori Kuwana, Kozo Sekimoto, Kozo Saito, and Forman A. Williams. Scaling fire whirls. Fire Safety J., 43(4):252 - 257, 2008.

23 Kang Yong Lee, Seungeun Park, Young Rang Lee, and Sang Kug Chung. Magnetic droplet microfluidic system incorporated with acoustic excitation for mixing enhancement. Sensor. Actuat. A Phys., 243:59 - 65, 2016.

24 Chao-Yaug Liao, Michel Bouriauand, Patrice L. Baldeck, JeanClaude Leon, Cedric Masclet, and Tien-Tung Chung. Twodimensional slicing method to speed up the fabrication of micro-objects based on two-photon polymerization. Applied Physics Letters, 91(3):033108, 2007.
25 N. Liron and S. Mochon. Stokes flow for a stokeslet between two parallel flat plates. J. Eng. Math, 10(4):287-303, 1976.

26 P. Marmottant and S Hilgenfeldt. Controlled vesicle deformation and lysis by single oscillating bubbles. Nature, 453:153156, 2003.

27 R. B. Maxwell, A. L. Gerhardt, M. Toner, M. L. Gray, and M. A. Schmidt. A microbubble-powered bioparticle actuator. J. Microelectromech. S., 12(5):630-640, Oct 2003.

28 F. Mekki-Berrada, T. Combriat, P. Thibault, and P. Marmottant. Interactions enhance the acoustic streaming around flattened microfluidic bubbles. J. Fluid Mech., 797:851-873, 0062016.

29 Flore Mekki-Berrada, Pierre Thibault, and Philippe Marmottant. Acoustic pulsation of a microbubble confined between elastic walls. Phys. Fluids, 28(3):032004, 2016.

30 M. Minnaert. Xvi. on musical air-bubbles and the sounds of running water. M. Philos. Mag., 16(104):235-248, 1933.

31 D. Olvera-Trejo and L. F. Velasquez-Garcia. Additively manufactured mems multiplexed coaxial electrospray sources for high-throughput, uniform generation of core-shell microparticles. Lab Chip, 16:4121-4132, 2016.

32 Julio M. Ottino and Stephen Wiggins. Introduction: mixing in microfluidics. Philos. T. Roy. Soc. A, 362(1818):923-935, 2004.

33 P.K. Panigrahi. Transport Phenomena in Microfluidic Systems. Wiley, 2016.

34 Brian Seed. Silanizing Glassware. John Wiley and Sons Inc., 2001.

35 T. A. Spelman and E. Lauga. Arbitrary axisymmetric steady streaming: Flow, force and propulsion. J. Eng. Math, pages 1-35, 2016.

36 Todd M. Squires and Stephen R. Quake. Microfluidics: Fluid physics at the nanoliter scale. Rev. Mod. Phys., 77:977-1026, Oct 2005.

37 Abraham D. Stroock, Stephan K. W. Dertinger, Armand Ajdari, Igor Mezić, Howard A. Stone, and George M. Whitesides. Chaotic mixer for microchannels. Science, 295(5555):647651, 2002.

38 Yong Kweon Suh and Sangmo Kang. A review on mixing in microfluidics. Micromachines, 1(3):82-111, Sep 2010.

39 Geoffrey Taylor. Dispersion of soluble matter in solvent flowing slowly through a tube. P. Roy. Soc. Lond. A. Mat., 219(1137):186-203, 1953.

40 C. Wang, S. V. Jalikop, and S. Hilgenfeldt. Efficient manipulation of microparticles in bubble streaming flows. Biomicrofluidics, 6(1):012801, 2012. 
41 C. Wang, B. Rallabandi, and S. Hilgenfeldt. Frequency dependence and frequency control of microbubble streaming flows. Phys. Fluids, 25(2):022002, 2013.
42 S Wang, X Huang, and C Yang. Microfluidic bubble generation by acoustic field for mixing enhancement. J. Heat Transfer, 134(5):051014, 2012. 